\title{
SCANNING TUNNELING MICROSCOPY/SPECTROSCOPY INVESTIGATIONS OF THE INTERFERENCE EFFECTS CAUSED BY THE CIRCULAR PITS ON THE THERMALLY OXIDIZED GRAPHITE SURFACE
}

\author{
Z. KluseK*, M.W. Kalinowski, W. Olejniczak and P. Kobierski \\ Department of Solid State Physics, University of Łódź \\ Pomorska 149/153, Łódź 90-236, Poland
}

\begin{abstract}
The thermal oxidation of the graphite leads to the removal of monoatomic carbon layers from the surface and formation of circular pits on the exposed plane. Near the pit edges the scanning tunneling spectroscopy measurements show a series of very narrow flat regions on the $I / V$ characteristics. The observed $I / V$ flat regions appear only when the characteristic is recorded very close to the pit edges. The appearance of tunneling current steps can be explained by the oscillating character of the local density of states near the pit edges. A simple quantum mechanical model in $2 \mathrm{D}$ based on boundary condition for an electron wave function is proposed.
\end{abstract}

PACS numbers: 33.60.Fy, 61.16.Ch, 71.20.-b

\section{Introduction}

The first-principles band calculation performed on the stepped surface of graphite showed existence of a localized state at the Fermi level [1]. This state is due to the cut of graphite sheet and is localized near the edges. The step acts as a potential barrier. In such a case additional process can be observed: incident surface Bloch wave can be reflected and will interfere with the incident wave leading to the oscillatory density of states. In order to detect the oscillations of electron local density of states (LDOS) near the step edges one should use a specific surface preparation and techniques which provide local spectroscopic information at an atomic level.

\footnotetext{
* Corresponding author.
} 


\section{Experimental}

A fresh surface of highly oriented pyrolytic graphite (HOPG) was prepared by cleavage with adhesive tape before thermal oxidation. The oxidation process was carried out in an externally heated quartz tube in ambient air conditions. In order to obtain well defined circular pits different temperatures and etching times were applied. The scanning tunneling microscopy/spectroscopy (STM/STS) results were obtained using a home-built apparatus with tunneling spectroscopic facilities. Experimental details are presented elsewhere [2-6].

\section{Results and discussion}

\subsection{Scanning tunneling microscopy results}

In Fig. 1A typical image of etched pits on an HOPG sample treated at $800^{\circ} \mathrm{C}$ in air for $30 \mathrm{~min}$ is shown. The growth of the etch pits causes erosion of the top layer. Due to further oxidation a surface with several levels of atomically flat terraces is formed. The topography presented exhibits up to two levels of atomic terraces with different radii of curvature. Circular pits randomly distributed over the terraces are also found. The depth of the etched pits can be estimated from the height profile. The average value is $0.35 \mathrm{~nm}$, which is approximately the spacing between graphite layers. Double layer pits are also observed. Triangular atomic structures at the bottom of the pit and on the top plane close to the pit edges can

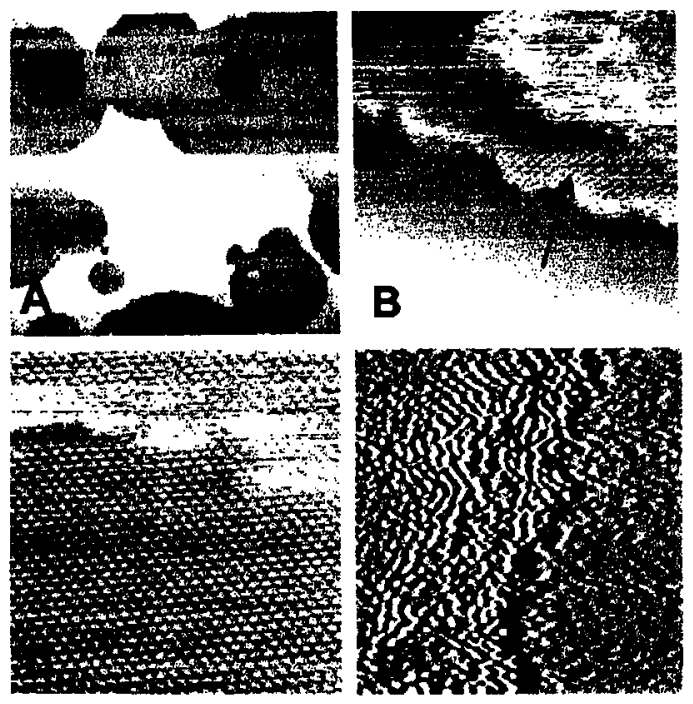

Fig. 1. (A) $600 \mathrm{~nm} \times 600 \mathrm{~nm}$ STM image of the thermally etched graphite at $800^{\circ} \mathrm{C}$ for $30 \mathrm{~min}$. (B) $17 \mathrm{~nm} \times 17 \mathrm{~nm} \mathrm{STM}$ image of the pit edges where $(\sqrt{3} \times \sqrt{3}) R 30^{\circ}$ superstructure appears (indicated by an arrow). (C) $8 \mathrm{~nm} \times 8 \mathrm{~nm}$ regions near the monolayer pit edges where a typical triangular structure of graphite with an extra contrast is resolved. (D) $9 \mathrm{~nm} \times 9 \mathrm{~nm}$ region where an interference effect is observed. 
be resolved. However, the $2-4 \mathrm{~nm}$ region near the edges of the pit is different from that in the smooth areas on the basal plane. In these regions a superstructure with the periodicity of $(\sqrt{3} \times \sqrt{3}) a(a=0.246 \mathrm{~nm})$ and rotated $30^{\circ}$ degrees with respect to the underlying graphite lattice is often observed (Fig. 1B). Similar structures can be seen on graphite near defects shaped like steps or adsorbed metal particles. Different theoretical models are used to explain the observed picture [7-9]. It seems that the observed superstructures have electronic nature and do not show real surface reconstruction. Apart from the well-known $(\sqrt{3} \times \sqrt{3}) R 30^{\circ}$ superstructure, a relatively unperturbed surface near the pit edges is also observed, see Fig. $1 \mathrm{C}$.

Another intriguing structure is prescnted in Fig. 1D, where the upper terrace near the pit edges consists of several bright lines running parallel to the edges. In this case we expect that such oscillations are due to the interference between the incident and reflected electron waves at the step. The density of state oscillation period is about $0.25 \mathrm{~nm}$ and falls off with increasing distance from the step. This image is very similar to the one obtained for $\mathrm{Au}(111)$ and $\mathrm{Ag}(111)$ surfaces with steps, where an oscillation of electron density of states is observed [10].

\subsection{Scanning tunneling spectroscopy results}

The next stage in our investigations is to measure the tunneling spectra in the regions where different superstructures appear. In Fig. 2 the $I / V$ data for the superstructure presented in Fig. 1D is shown. The three curves, denoted as $\alpha, \beta$, and $\gamma$, are recorded at different distances from the pit edges. The curve $\alpha$ is recorded at about $5 \mathrm{~nm}$ from the pit step, while the curve $\gamma$ at about $1 \mathrm{~nm}$ from the pit step. The curve denoted as $\beta$ is recorded at the intermediate distance. All these $I / V$ curves show the same asymmetric shape, which is typical of pure graphite $[5,6,11,12]$. However, it is not difficult to observe some differences in

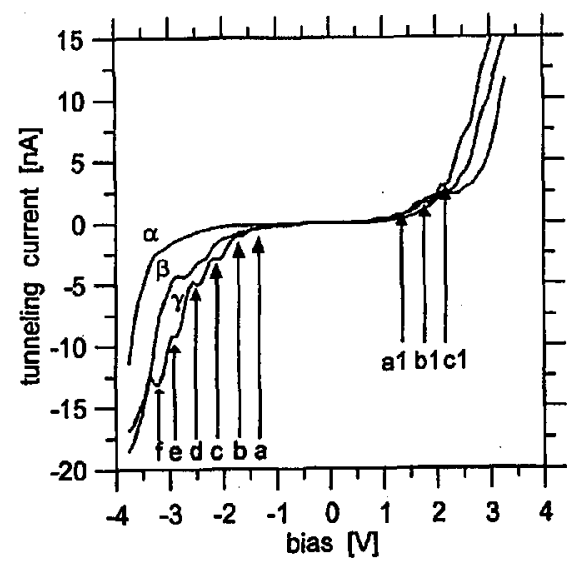

Fig. 2. Different current-voltage curves recorded over the superstructure presented in Fig. 1D. The curve $\alpha$ has been recorded at about $5 \mathrm{~nm}$, while the curve $\gamma$ at about $1 \mathrm{~nm}$ from the pit step. The curve denoted as $\beta$ was recorded at an intermediate distance. The arrows indicate narrow flat regions on the $\gamma I / V$ curve. 
comparison to basal plane of graphite spectra. In the curve $\gamma$ one observes a series of very narrow flat regions $(a, b, c, d, e, f, a 1, b 1, c 1)$ that should manifest themselves as prominent dips in the normalized conductance $(\mathrm{d} I / \mathrm{d} V) /(I / V)$. In the investigated bias range, the dips in $(\mathrm{d} I / \mathrm{d} V) /(I / V)$ appear equally spaced with a splitting of approximately $0.5 \mathrm{eV}$. The observed $I / V$ flat regions appear only when the characteristics is recorded very close to the pit edges. In our interpretation the appearance of tunneling current steps can be explained by the oscillating character of the LDOS near the pit edges. It is worth pointing out that in the case of the $(\sqrt{3} \times \sqrt{3}) R 30^{\circ}$ superstructure (Fig. 1B), the STS measurements do not show substantial differences in comparison to basal plane of graphite. A typical $I / V$ curve taken over this superstructure resembles the curve $\alpha$ presented in Fig. 2. In order to explain experimental results we consider the following quantum-mechanical (QM) problem reducible to $2 \mathrm{D}$ :

$$
\psi(x, y, z)=\psi_{0}(x, y) \mathrm{e}^{-\alpha^{2} z} .
$$

The exponential decay in $z$ direction means that electrons are confined to the surface. In $2 \mathrm{D}$ we simply have

$$
H \psi_{0}=\widehat{E} \psi_{0}, \quad \widehat{E}=E+\frac{\hbar^{2} \alpha^{4}}{2 m^{*}}, \quad H=-\frac{\hbar^{2}}{2 m^{*}} \nabla^{2}+V,
$$

$m^{*}$ is an effective mass of an electron and $\widehat{E}$ is an energy of an electron, $\nabla^{2}$ is a 2D Laplace operator and $V$ is a potential describing the pit on the surface. For simplicity we propose that $V$ is infinite inside the pit and zero outside. Thus

$$
V(x, y)=\widehat{V}(r, \phi)=\left\{\begin{array}{cc}
\infty, & r \leq a, \\
0, & r>a .
\end{array}\right.
$$

It is obvious that for $\psi_{0}=0$ for $r \leq a$ ( $a$ - radius of the pit). One simply obtains

$$
\widehat{\psi}_{0}(x, y)=\psi_{0}(r, \phi)=\frac{A}{\sqrt{2 \pi}} e^{\mathrm{i} m \phi} J_{m}(k r)
$$

where $m=0, \pm 1, \pm 2, \ldots$, and $J_{m}$ is a Bessel function, $A=$ const, and .

$$
k^{2}=\frac{2 m^{*}\left(E+\frac{\hbar^{2} \alpha^{4}}{2 m^{*}}\right)}{\hbar^{2}} .
$$

Let $\zeta_{m n}$ be a zero of Bessel function $J_{m}, J_{m}\left(\zeta_{m n}\right)=0$. Thus one obtains

$$
E=E_{m n}=\frac{\hbar^{2}}{2 m^{*} a^{2}}\left(\zeta_{m n}^{2}-\alpha^{4} a^{2}\right)
$$

The oscillatory pattern on the STM images and the flat regions on the STS characteristics are coming from the shape of the wave function $\psi_{0}$. It is due to the fact that in the STM/STS techniques the tunneling current is given by

$$
I=\int_{E_{f}}^{E_{f}+e V} \rho_{\mathrm{S}}(r, E) \rho_{\mathrm{T}}(r, E-e V)[f(E-e V)-f(E)] T(r, E, e V) \mathrm{d} E,
$$

where $T$ is the transmission coefficient, $f(E)-$ Fermi function, $\rho_{\mathrm{S}}$ and $\rho_{\mathrm{T}}$ are the density of states of a sample and a tip at a location $r=(x, y)$. The energy $E$ is measured with respect to the bottom of the conductance band of the negative 
biased sample. The LDOS of the sample $\rho_{\mathrm{S}}(E)$, which is probed by STM/STS techniques equals to

$$
\rho_{\mathrm{S}}(E, x, y) \propto\left|J_{m}(k r)\right|^{2}=J_{m}^{2}\left(\frac{\zeta_{m n} r}{a}\right)=J_{m}^{2}\left(\frac{\zeta_{m, n} \sqrt{x^{2}+y^{2}}}{a}\right),
$$

where $E$ is given by the formula (6). Moreover, we should take under consideration an influence of crystalline field induced by the graphite lattice on an electron. Thus the oscillatory pattern can be seen only up to some distance not to be large. For the density of state oscillation goes down with an increase in the distance from the step edges, we cannot observe such effects on the curve $\alpha(5 \mathrm{~nm}$ far from the pit edges). Here it should be noted that in the areas close to the pit edges the surface heterogroups $(\mathrm{C}-\mathrm{OH}, \mathrm{C}-\mathrm{OOH})$ appear due to thermal oxidation and could result in Coulomb blockade effect [12]. It means that not only interference effect but also chemical composition on the surface can be responsible for observation of series of very narrow flat regions on the $I / V$ characteristics. More research is needed to understand the influence of local surface defects on tunneling spectra. Further experiments are in progress.

\section{Conclusions}

We have observed oscillating pattern on the STM images and very narrow flat regions on the STS current-voltage characteristics taken near the circular pit edges on the thermally oxidized HOPG (0001) basal plane. By analyzing an influence of step edges on graphite LDOS we came to the conclusion that we are dealing with structure different from Kobayashi [1] localized surface state which appears as a maximum of LDOS near the Fermi level. An oscillation character of LDOS (due to interference effect caused by boundary conditions in QM problem reducible to 2D) or Coulomb blockade are assumed to explain the observed phenomena.

\section{Acknowledgments}

The work was supported by the Committee for Scientific Research (Poland) grant No. 7 T08C 04211.

\section{References}

[1] K. Kobayashi, Phys. Rev. B 48, 1757 (1993).

[2] H. Chang, A.J. Bard, J. Am. Chem. Soc. 112, 4598 (1990).

[3] H. Chang, A.J. Bard, J. Am. Chem. Soc. 113, 5588 (1991).

[4] G. Binnig, H. Rohrer, IBM J. Res. Dev. 30, 355 (1986).

[5] Z. Klusek, Appl. Surf. Sci. 108, 405 (1997).

[6] Z. Klusek, Acta Phys. Pol. A 91, 569 (1997).

[7] H.A. Mizes, J.S. Foster, Science 244, 559 (1989).

[8] J. Valenzuela-Benavides, L. Morales de la Garza, Surf. Sci. 330, 227 (1995).

[9] J. Xhie, K. Sattler, U. Muller, N. Venkateswarn, G. Raina, Phys. Rev. B 43, 8917 (1991).

[10] Ph. Avouris, I.W. Lyo, R.E. Walkup, J. Vac. Sci. Technol. B 12, 1447 (1994). 
[11] R.J. Colton, S.M. Baker, R.J. Driscoll, M.G. Youngquist, J.D. Baldeschwieler, W.J. Kaiser, J. Vac. Sci. Technol. A 6, 349 (1988).

[12] N. Venkateswaran, K. Sattler, U. Muller, B. Kaiser, G. Raina, J. Xhie, J. Vac. Sci. Technol. B 9, 1052 (1991). 